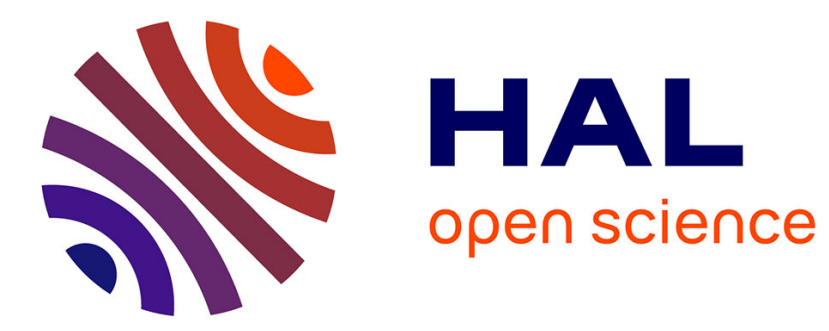

\title{
Reaction kinetics of non-localised particle-trap complexes
}

Alexander V Barashev, Stanislav I Golubov, Yuri Osetsky, Roger E Stoller

\section{To cite this version:}

Alexander V Barashev, Stanislav I Golubov, Yuri Osetsky, Roger E Stoller. Reaction kinetics of non-localised particle-trap complexes. Philosophical Magazine, 2010, 90 (07-08), pp.897-906. 10.1080/14786430903190825 . hal-00581025

\section{HAL Id: hal-00581025 \\ https://hal.science/hal-00581025}

Submitted on 30 Mar 2011

HAL is a multi-disciplinary open access archive for the deposit and dissemination of scientific research documents, whether they are published or not. The documents may come from teaching and research institutions in France or abroad, or from public or private research centers.
L'archive ouverte pluridisciplinaire HAL, est destinée au dépôt et à la diffusion de documents scientifiques de niveau recherche, publiés ou non, émanant des établissements d'enseignement et de recherche français ou étrangers, des laboratoires publics ou privés. 


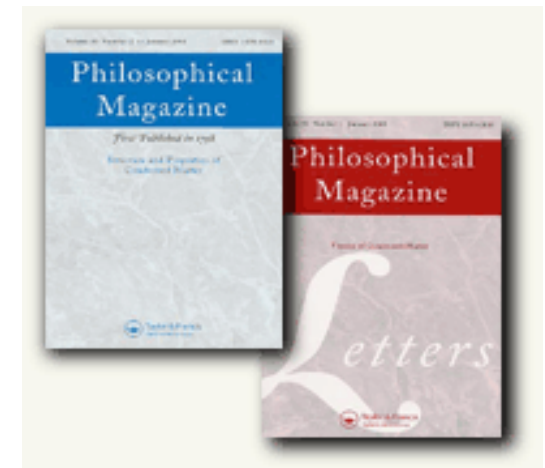

\section{Reaction kinetics of non-localised particle-trap complexes}

\begin{tabular}{|r|l|}
\hline Journal: & Philosophical Magazine \& Philosophical Magazine Letters \\
\hline Manuscript ID: & TPHM-09-Apr-0176.R1 \\
\hline Journal Selection: & Philosophical Magazine \\
\hline Date Submitted by the & 15 -Jul-2009 \\
\hline Complete List of Authors: & $\begin{array}{l}\text { Barashev, Alexander; The University of Liverpool, Engineering } \\
\text { Golubov, Stanislav; University of Tennessee, Center for Materials } \\
\text { Processing; ORNL, Materials Science and Technology Division } \\
\text { Osetsky, Yuri; ORNL, Materials Science and Technology Division } \\
\text { Stoller, Roger; Oak Ridge National Laboratory, Metals and Ceramics } \\
\text { Division }\end{array}$ \\
\hline Keywords: & diffusion, kinetics of reactions \\
\hline Keywords (user supplied): & transition state theory, dissociation reactions, edge dislocations \\
\hline
\end{tabular}

\section{s ScholarONE" \\ Manuscript Central}




\title{
Reaction kinetics of non-localised particle-trap complexes
}

\author{
A.V. Barashev†*, S.I. Golubov $§$, Yu.N. Osetsky $\ddagger$ and R.E. Stoller $\ddagger$ \\ $\dagger$ Department of Engineering, The University of Liverpool, \\ Brownlow Hill, Liverpool, L69 3GH, UK \\ ¥Materials Science and Technology Division, ORNL, \\ Oak Ridge, TN 37831- 6138, USA \\ $\S$ Center for Materials Processing, University of Tennessee, \\ East Stadium Hall, Knoxville, TN 37996-0750, USA
}

\begin{abstract}
The classical transition state theory for calculating complex dissociation rates requires separation of time scales. It is shown here that this condition is satisfied for complexes with long-range interaction, such as of an edge dislocation and a cluster of self-interstitial atoms in metallic materials, and, hence, one can apply the equations for first-order reactions with a rescaled mean dissociation time and the cross-section of complex formation. The rescaling coefficient is the Eyring transmission coefficient. A general expression for this coefficient through the first two moments of the distribution function of dissociation times is derived. It is shown that it is equal to unity if the dividing surface between 'bound' and 'free' states is defined as that where the interaction energy is equal to the thermal energy.
\end{abstract}

Key words: transition state theory, dissociation reactions, complexes, edge dislocations, metals.

*Author for correspondence. Email: a.barashev@liv.ac.uk; Tel.: +44 1517945773. 


\section{Introduction}

Neutron irradiation of metallic materials initiates cascades of atomic displacements, which produce the primary damage in the form of point defects, vacancies and self-interstitial atoms (SIAs), and their clusters [1-5]. The SIA clusters are highly mobile small dislocation loops, which migrate one-dimensionally along the close-packed directions of their Burgers vectors [2,6-9]. They are observed to decorate edge dislocations in metals such as $\mathrm{Ni}$ and $\mathrm{Mo}$ $[10,11]$. As a result, the dislocations become pinned and the materials brittle. The decoration is thus one of the key processes in the microstructure evolution in metallic materials under cascade irradiation, but is still understood poorly. One of the obstacles is the lack of understanding of the kinetics of the interaction between the interstitial clusters and dislocations. A complexity originates from the long interaction range: e.g. at $600 \mathrm{~K}$, the distance from the core of an edge dislocation, at which the interaction energy is of the order of the thermal energy, is $\sim 50 \mathrm{~nm}$ (for a cluster of 10 SIAs), that is $\sim 200$ Burgers vector lengths. This results in a significantly non-exponential distribution of dissociation times, which may destroy applicability of the reaction rate and transition state theories, which generally have broad applications in chemistry and physics [12].

In the mean-field approach of the chemical-reaction theory [13-15], the evolution of the concentration of bi-molecular A-B complexes, $N(t)$, is described by an equation which includes reaction rates for complex formation, $F(t)$, and dissociation, $D(t)$ :

$$
\dot{N}(t)=F(t)-D(t) \text {. }
$$

It is usually coupled with equations for the concentrations of unpaired particles, $C_{\mathrm{A}}(t)$ and $C_{\mathrm{B}}(t)$, which define the formation rate: $F(t)=\alpha C_{\mathrm{A}}(t) C_{\mathrm{B}}(t)$. The rate coefficient $\alpha$ is found using available methods [14]; e.g. for diffusion-controlled coagulation $\alpha \propto 4 \pi r D_{\mathrm{AB}}$, where $r$ and $D_{\mathrm{AB}}$ are the total radius and diffusion coefficient of $\mathrm{A}$ and $\mathrm{B}$ species, which is the classical result of Smoluchowski [16]. Determining the dissociation rate requires utilising ideas of the seminal transition-state theory [13,15] and the principle of microscopic reversibility (detailed balance) for chemical equilibrium [17]. The result depends on the probability function of dissociation times, $P(t)$, i.e. the probability that an event occurs before time $t$. For a random process without memory, $P(t)=1-\exp (-t / \tau)$ with the mean 


\section{Analysis}

Consider a system under what may be non-equilibrium conditions. such conditions: accumulation of complexes with a nonzero formation rate and annealing from some initial state with nonzero concentration of complexes. The former process is more general, since it involves an extra process of complex formation and is considered below. It will be shown, though, that to a first approximation the final expression for the dissociation rate can be written in general, independent of the process, form, which is applicable to the annealing problem as well. Then, $N(t)$ is given by an integral accounting for all complexes formed and survived by a time $t$, and $D(t)$ by a similar integral for complexes dissociating at this instant of time:

$$
N(t)=\int_{0}^{\infty} d s F(t-s)[1-P(s)],
$$




$$
D(t)=\int_{0}^{\infty} d s F(t-s) P^{\prime}(s)
$$

For an exponential distribution, $P^{\prime}(t)=\tau^{-1}[1-P(t)]$ and equation (2) follows from equations (3) and (4) immediately. In general, one can obtain an equation for $D(t)$ as a series of progressively higher order $n$ time derivatives of $N(t)$ by representing $F(t)$ as a Taylor series around time $t$ :

$$
F(t-s)=\sum_{n=0}^{\infty}(-1)^{n} \frac{s^{n} F^{(n)}(t)}{n !} .
$$

Then, from equations (3) and (4), one obtains that

$$
\begin{aligned}
& N(t)=\sum_{n=0}^{\infty}(-1)^{n} \frac{\left\langle t^{n+1}\right\rangle F^{(n)}(t)}{(n+1) !} \\
& D(t)=\sum_{n=0}^{\infty}(-1)^{n} \frac{\left\langle t^{n}\right\rangle F^{(n)}(t)}{n !} .
\end{aligned}
$$

By differentiation of equation (6) $n$ times one can obtain the following expressions for the derivatives of $F(t)$ :

$$
\tau F^{(n)}(t)=N^{(n)}(t)-\sum_{k=1}^{\infty}(-1)^{k} \frac{\left\langle t^{k+1}\right\rangle F^{(n+k)}(t)}{(k+1) !} .
$$

By substituting equation (8) with increasing $n$ into equation (7) and by treating the deviation from an exponential distribution as a perturbation, hence neglecting high order time derivatives of $F(t)$, one obtains the dissociation rate as a series of progressively higher order, $n$, time derivatives of $N(t)$. For $n=0$, one reproduces equation (2) and, for $n=1$, the first order time derivative enters the equation:

$$
D(t)=N(t) / \tau+\gamma \dot{N}(t)
$$


By substituting equation (9) into equation (1), one obtains the rate equation for the concentration of complexes in its conventional form as for the exponential distribution but with a rescaled mean dissociation time, $\tilde{\tau}$, and a cross section for complex formation, $\tilde{\alpha}$ $(F \propto \alpha$, hence $\tilde{F}=F \& / d \alpha)$ :

$$
\begin{aligned}
& \dot{N}(t)=\tilde{F}(t)-N(t) / \tilde{\tau}, \\
& \tilde{\tau}=\tau \kappa^{-1}, \tilde{\alpha}=\alpha \kappa, \\
& \kappa=(1+\gamma)^{-1}=2 \tau^{2} /\left\langle t^{2}\right\rangle .
\end{aligned}
$$

Hence, the evolution of complex concentration with a non-exponential distribution of dissociation times is characterised by the same equilibrium state as for an exponential distribution with the same mean value but with a different characteristic time for approaching this state. 
The applicability limits of equations (9) and (10) can be estimated as those when the absolute value of the term for $n=2$, which is $\tau\left[(1+\gamma)^{2}-\delta\right] \ddot{N}(t)$, where $\delta=\left\langle t^{3}\right\rangle / 6 \tau^{3}$, is much smaller than that of the term with the first order time derivative, $\gamma \dot{N}(t)$ :

$$
\left|\delta \kappa^{2}-1\right|<<|\kappa-1| \Theta(t)
$$

where $\Theta(t)=|\dot{N} / \tilde{\tau} \ddot{N}|$, which is equal to unity for the exponential decay.

Equation (10) describes the exponential decay law. Hence, the rescaling can be viewed as a redefinition of the complex concentration that eliminates particles that do not follow the exponential distribution. Generally, the lowest energy must define the rescaled dissociation time, since the slowest process must govern the transient processes. Therefore, the new definition should limit complex formation events to those which occur when a migrating particle visits a site of the lowest interaction (highest binding) energy. Such a scenario is exactly that of the transition state theory. Hence, the factor $\kappa$ in equation (12) has the meaning of the Eyring transmission coefficient [13,15] for complex formation rate and must be equal to the fraction of particles that reach the deep regions of a well and correct for reactive trajectories that re-cross the transition state and return without 'decomposition' (of a 'free' state) and formation of a 'bound' state. (Evidently, for the diffusion-controlled reactions, the transmission factor for the dissociation rate is equal to $\alpha \tau_{\mathrm{j}}$, where $\tau_{\mathrm{j}}$ is the mean time between jumps in perfect crystal. Hence $\kappa \alpha$ is a common factor for both the formation and dissociation rates.)

To verify the validity of this analysis, we performed Monte Carlo (MC) simulations of particle diffusion in a system containing a 1-D triangular potential well of depth $E$, defined on an interval $x \in\left[-x_{\text {max }}, x_{\max }\right]$ :

$$
U(x)=-E\left(1-|x| / x_{\max }\right)
$$

\section{In these calculations, the particle diffused in the system $x \in\left[0, x_{\max }\right]$ with 'mirror' boundary}

conditions. The uphill and downhill jump frequencies, $v^{+}$and $v^{-}$, were calculated as $v^{ \pm}=\exp \left(\mp \beta E a_{\mathrm{j}} / 2 x_{\max }\right) / \tau_{\mathrm{j}}$, where $\beta^{-1}=k_{\mathrm{B}} T, k_{\mathrm{B}}$ being the Boltzmann constant and $T$ the 
temperature, and $a_{\mathrm{j}}$ is the jump distance. The value $<t>=\left(v^{+}+v^{+}\right)^{-1}$ is the mean waiting time before a jump. The calculations were performed for wells of different width $(50,100$ and $200 a_{\mathrm{j}}$ ) and depth $(\beta E=3.87$ and 7.74, which correspond to $E=0.1$ and $0.2 \mathrm{eV}$, respectively, at $T=300 \mathrm{~K})$ and for statistics of $\sim 500,000$ dissociation events in each case. Naturally, an event of complex formation is defined by the jump of a particle into the well and a dissociation event by a reverse jump. In this case, the calculations of the mean dissociation time for wide wells $\left(x_{\max }>>a_{\mathrm{j}} \beta E\right)$ reproduce well the following equation derived by considering the partition function of the canonical ensemble [18]:

$$
\tau_{1-\mathrm{D} \mathrm{tri}} \approx 2 \tau_{\mathrm{j}}\left(x_{\text {max }} / a_{\mathrm{j}} \beta E\right)[\exp (\beta E)-1] .
$$

The calculation results for $1-P(t)$, the so-called survival function, are shown in Figure 1 for wells of different width and depth. The range of well width was chosen to represent 1-D migration of an interstitial loop in the stress-field of an edge dislocation [11]. The error bars are small and not visible on the graph. The function $1-P(t)$ is shown to decrease steeply at short times, while an exponential dependence evolves at long times. The deviations from the exponential distribution are quite large. For example, consider the curve calculated for $\beta E=3.87$ and $x_{\max }=100 a_{\mathrm{j}}$. At $t=\tau$, the number of complexes is reduced by $\sim 25$ times, almost an order of magnitude higher than $e$ for the exponential distribution. The variance of this distribution, $\sigma_{t}$, is about seven times larger than for the corresponding exponential one. One calculation for a square well (labelled 'Square') is also shown in Figure 1 and it demonstrates a dependence that is close to exponential decay. This is because any square well is characterised by single energy, in contrast to triangular well, in which depth of the well is not constant. In other words the square wells do not contain high-energy states (shallow regions) inside the well, from where a particle can dissociate without visiting deep regions. (Square wells may also demonstrate deviations from the exponential decay for sufficiently wide wells, such that the diffusion time of a particle over distances of the order of well width are comparable with the mean dissociation time from the well.)

[Insert Figure 1 about here] 
The curve for $\beta E=3.87$ and $x_{\max }=50 a_{\mathrm{j}}$, which is labelled ' $x=0$ ', is the distribution of dissociation times from the bottom of the well, that is, when all trajectories of diffusing particles started from $x=0$, the deepest position in the well. In this calculation, the mean dissociation time was $\sim 15000 \tau_{\mathrm{j}}$, more than an order of magnitude longer than $1200 \tau_{\mathrm{j}}$, which is obtained from equation (15) and the corresponding MC calculation. This compares well with an equation for wide wells derived by the flux-over-population method pioneered by Farkas [19] (see Appendix A):

$$
\tilde{\tau}_{1-\mathrm{D} \text { tri }} \approx 2 \tau_{\mathrm{j}}\left(x_{\max } / a_{\mathrm{j}} \beta E\right)^{2}[\exp (\beta E)-1-\beta E]
$$

In addition, this calculation demonstrates an exponential decay, which indicates that the deviation from the exponential function is due to trajectories which do not reach the bottom of the well. Thus, by redefining the complex formation event as that occurring when a particle passes through the deepest point of the well, one restores the exponential distribution. This is because the work required to remove a particle from this point is unique.

According to our interpretation of rescaling, the factor $\kappa^{-1}$ should be equal to the ratio of mean dissociation times given by the two definitions of complex creation events: (1) as a jump through $x=0$, the deepest point of the well, and (2) as a jump over the edge of the well, $x=x_{\max }$. A comparison of $\kappa^{-1}$ with this ratio calculated by MC is given in Figure 2. As seen, $\kappa^{-1}$ is high for wide wells, but the redefinition of complex formation makes it close to unity. Before the redefinition, it compares well with the ratio of times, which confirms the interpretation. Note that the calculations suggest the following equation, which satisfies the limiting case of a nil effect for a single-site trap (at $x_{\max }=a_{\mathrm{j}}$ ) and is equal to the ratio of equations (15) and (16) for wide wells:

$$
\kappa_{1-\mathrm{D} \text { tri }}^{-1} \approx 1+\left(x_{\max } / a_{\mathrm{j}}-1\right)(\beta E)^{-1}[1-\beta E \exp (-\beta E)]
$$

where the factor in square brackets is close to unity. As seen from Figure 2, equation (17) is in agreement with the MC calculations over the entire range of the potential well width used. It also shows that $\kappa^{-1}$ is higher than unity even for narrow wells, which may be important. 
[Insert Figure 2 about here]

We verified how the rescaling operates for the accumulation of complexes of diffusing particles with a 1-D triangular well at a constant formation rate, $F$. For this, we calculated $P(t)$ by MC and performed the integration indicated in equation (3). The results are presented in Figure 3 (on the left) for $x_{\max } / a_{\mathrm{j}}=20$ and $\beta E=3.87$, where the number of complexes is normalised by the steady-state value $N_{\max }=\tau F$. The data on the right are for the annealing of the same complexes. As seen in the figure, the characteristic time for approaching the steady-state concentration is higher by a factor of $\kappa^{-1} \approx 6$ as compared with the exponential dependence for a single-site trap with the same mean dissociation time, $p(t, \tau)=1-\exp (-t / \tau)$. The solution of equation (10) $p(t, \kappa \tau)$ coincides with the MC calculations everywhere but at short times. The deviation is due to particles that reach only shallow regions of the well, of the order of thermal energy: $U\left(x_{\max }-\tilde{x}\right) \approx-k_{\mathrm{B}} T$, $\tilde{x}=x_{\max } / \beta E$, before dissociation with the mean time $\tau^{\text {th }} \approx \tau_{\mathrm{j}} \tilde{x} / a_{\mathrm{j}}$. Indeed, as seen from Figure 3, the function $p(t, \kappa \tau)+p\left(t, \tau^{\text {th }}\right) \tau^{\text {th }} / \tau$, which is a superposition of the two processes, is close to the MC calculations.

[Insert Figure 3 about here]

The same conclusion can be reached by considering diffusion in a potential field with a sink at $x=0$. It can be shown that the same rate of particle absorption is achieved in the absence of the interaction field but for a sink at $x=\tilde{x}$, where the interaction energy is $-k_{\mathrm{B}} T$ (see Appendix B). Hence, particles passing beyond this energy level have a high probability of reaching the bottom of the well. Since we know from our calculations that dissociation of particles from the bottom of the well obeys exponential distribution, the condition $x=\tilde{x}$ should define a point of no return, hence such new well boundary, for which the distribution of dissociation times is exponential. The result is almost trivial. Indeed, the condition for the interaction energy between a migrating point defect (vacancy or interstitial atom) and an edge dislocation to be less than $k_{\mathrm{B}} T$ is commonly used as absorbing boundary conditions (see, e.g. [20]). And the assumption of point of no return is a prerequisite for the validity of the classical transition-state theory (see, e.g. [15], p.261). 
To understand why the rescaling works, consider a simplified representation of the distributions in Figure 1 as a superposition of two random processes with the mean times $\tau_{2}<<\tau_{1}: P^{\prime}(t)=\left[\exp \left(-t / \tau_{1}\right) / \tau_{1}+A \exp \left(-t / \tau_{2}\right) / \tau_{2}\right] /(1+A)$, where $A$ is a constant. Then, equation (13) reads $A \tau_{2}<<\tau_{1}$, i.e. it requires the number of complexes to be defined by particles passing through the deepest site of the well. In other words, the time-scales for escaping from deep and shallow regions must be well separated. The calculations in Figures 2 and 3 imply that this is valid despite the fact that such particles constitute only a small fraction of all particles visiting the trap region, i.e. $A>>1$. This is why $N(t)$ is defined by $\tau_{1}$, which is higher than the mean time by a factor of $1+A: \tau_{1} \approx\langle t\rangle(1+A)$. This factor can be obtained through the parameters of the distribution as $\kappa^{-1} \approx 1+A$, which explains the rescaling.

\section{Conclusions}

1. The dissociation of non-localised complexes can be described by the first-order kinetics as for random processes without memory. (The memory is transferred to the complex definition. Now the mere existence of a particle within the trap region is not enough to define a complex; for this, one should remember in addition whether it has visited a site of the maximum binding energy.) An amazing fact is that this remains valid even for very low values of $\kappa$, i.e. for large ratios of the rescaled and initial dissociation times, hence for such complexes as of an edge dislocation with an SIA cluster. This is due to the Arrhenius dependence of thermally-activated jumps, which makes the time-scales of escaping events from deep and shallow regions well separated.

2. A dividing surface between 'bound' and 'unbound' states defined as that where the interaction energy is equal to the thermal energy corresponds to the transmission factor equal to unity. Hence, particles that pass beyond this energy level with high probability reach the bottom of the well. This fact may be used in simulations to separate the timescales, e.g. of the fast diffusion of an SIA cluster in the matrix and slow dissociation from the region of strong interaction with edge dislocations. We note that, although this result has been obtained for triangular wells and thus needs 


\title{
Acknowledgments
}

\begin{abstract}
A.V.B. acknowledges a research grant from the UK Engineering and Physical Sciences Research Council. Research at ORNL was sponsored by the Division on Materials Sciences and Engineering (R.E.S. and Y.N.O.) and the Office of Fusion Energy Sciences (S.I.G.), U. S. Department of Energy, under contract no. DE-AC05-00OR22725 with UT-Battelle, LLC.
\end{abstract}




\section{Appendix A. The dissociation time of a particle from the bottom of the well}

Consider a stationary 1-D diffusion problem for particles migrating in potential $U(x)$ :

$$
\begin{aligned}
& j^{\prime}(x)=0, \\
& j(x)=-D C^{\prime}(x)-\beta D C(x) U^{\prime}(x), \\
& C\left(x_{\max }\right)=0, \\
& j(0)=a_{\mathrm{j}} / \tilde{\tau},
\end{aligned}
$$

where $j(x)$ is the flux, $C(x)$ is the concentration of particles and $D=a_{\mathrm{j}}^{2} / 2 \tau_{\mathrm{j}}$ is the diffusion coefficient. Equation (A3) defines an absorption boundary, thus providing only first passage particles through it. Equation (A4) defines a constant source of particles at $x=0$ with $\tilde{\tau}$ being the mean time between particle impingement. According to the flux-over-population method [19], $\tilde{\tau}$ is equal to the mean first passage time of particles from the bottom of the well through the well boundary, if on average only one particle is in the well region:

$$
\int_{\text {well }} d x C(r)=a_{\mathrm{j}}
$$

For a triangular potential well $U(x)=-E\left(1-x / x_{\text {max }}\right)$ defined on an interval $x \in\left[0, x_{\max }\right]$, the solution of equations (A1)-(A4) is given by

$$
C(x)=A\left\{\exp \left[B\left(x_{\max }-x\right)\right]-1\right\}
$$

where $A=2 \tau_{\mathrm{j}} x_{\max } / \tilde{\tau} a_{\mathrm{j}} \beta E$ and $B=\beta E / x_{\max }$. By substituting equation (A6) into equation (A5) one obtains the mean dissociation time $\tilde{\tau}_{1-\mathrm{D} \text { tri }}$ as

$$
\tilde{\tau}_{1-\mathrm{D} \mathrm{tri}} \approx 2 \tau_{\mathrm{j}}\left(\frac{x_{\max }}{a_{\mathrm{j}} \beta E}\right)^{2}[\exp (\beta E)-1-\beta E]
$$




\section{Appendix B. Annihilation of diffusing particles at a sink with long-range interaction}

Consider the same diffusion problem as in Appendix A, equations (A1) and (A2), but with different boundary conditions, defining complete absorption of migrating particles at the bottom of the well at $x=0$ :

$$
\begin{aligned}
& C(0)=0, \\
& C\left(x_{\text {max }}\right)=C_{0} .
\end{aligned}
$$

For the triangular potential well, equation (14), the solution is given by

$$
C(x)=C_{0} \frac{1-\exp \left(-E \beta x / x_{\max }\right)}{1-\exp (-E \beta)}
$$

The flux at $x=0$ is

$$
j(0)=-D C_{0} \beta E / x_{\max }[1-\exp (-E \beta)] \approx-D C_{0} \beta E / x_{\max } .
$$

In the absence of interaction field and with a sink at distance $x_{\max }-\tilde{x}$ (in other words, with complete absorption at this distance), the flux is

$$
j\left(x_{\max }-\tilde{x}\right)=-D C_{0} / \tilde{x}
$$

The two fluxes, equations (B4) and (B5), are equal to each other if

$$
\tilde{x}=x_{\max } / \beta E,
$$

for which

$$
U\left(x_{\max }-\tilde{x}\right)=-k_{\mathrm{B}} T .
$$


Thus, the same rate of particle absorption is achieved in the absence of the interaction field but with a sink at $x=\tilde{x}$, where the interaction energy is $-k_{\mathrm{B}} T$. We note that, although this $\underline{\text { result has been obtained for triangular wells, it is independent of the parameters defining well }}$ shape and, hence, should be general. 


\section{References}

[1] B.V. Guerard, D. Grasse and J. Peisl, Phys. Rev. Lett. 44 (1980) p.262.

[2] T. Diaz de la Rubia and M.W. Guinan, Phys. Rev. Lett. 66 (1991) p.2766.

[3] A.J.E. Foreman, W.J. Phythian and C. A. English, Philos. Mag. A 66 (1992) p.671.

[4] R.E. Stoller, J. Nucl. Mater. 233-237 (1996) p.999.

[5] D.J. Bacon, Yu.N. Osetsky, R. Stoller et al., J. Nucl. Mater. 323 (2003) p.152.

[6] Yu.N. Osetsky, D.J. Bacon, A. Serra et al., Philos. Mag. A 83 (2003) p.61.

[7] M. Kiritani, J. Nucl. Mater. 251 (1997) p.237.

[8] T. Hayashi, K. Fukmuto and H. Matsui, J. Nucl. Mater. 307-311 (2002) p.993.

[9] K. Arakawa, K. Ono, M. Isshiki et al., Science 318 (2007) p.956.

[10] B.N. Singh, A.J.E. Foreman and H. Trinkaus, J. Nucl. Mater. 249 (1997) p.91.

[11] H. Trinkaus, B.N. Singh and A.J.E. Foreman, J. Nucl. Mater. 249 (1997) p.103.

[12] G.R. Freeman (ed.), Kinetics of nonhomogeneous processes, (John Wiley and Sons, New York, 1987).

[13] H. Eyring, Chem. Phys. 3 (1935) p.107.

[14] D.F. Calef and J.M. Deutch, Ann. Rev. Phys. Chem. 34 (1983) p.493.

[15] P. Hanggi, P. Talkner and M. Borkovec, Rev. Mod. Phys. 62 (1990) p.251.

[16] M.V. Smoluchowski, Z. Phys. Chem. 92 (1917) p.192.

[17] R.C. Tolman, The Principles of Statistical Mechanics (Oxford University Press, London, UK, 1938).

[18] A.V. Barashev, S.I. Golubov, Yu.N. Osetsky et al., this issue.

[19] L. Farkas, Z. Phys. Chem. 125 (1927) p.236.

\section{[20] R.J. White, S.B. Fisher and P.T. Heald, Philos. Mag. 34 (1976) p.647.}




\section{Figure captions}

Figure 1. Probability that a dissociation event occurs after time $t$ for 1-D triangular wells of different width and depth, calculated by MC.

Figure 2. The dependence of $\kappa^{-1}$ and the ratio of mean dissociation times for two complex definitions on the interaction range calculated by $\mathrm{MC}$ results for 1-D triangular wells.

Figure 3. Time dependence of the number of complexes of migrating particles with 1-D triangular wells during steady complex formation and annealing. 


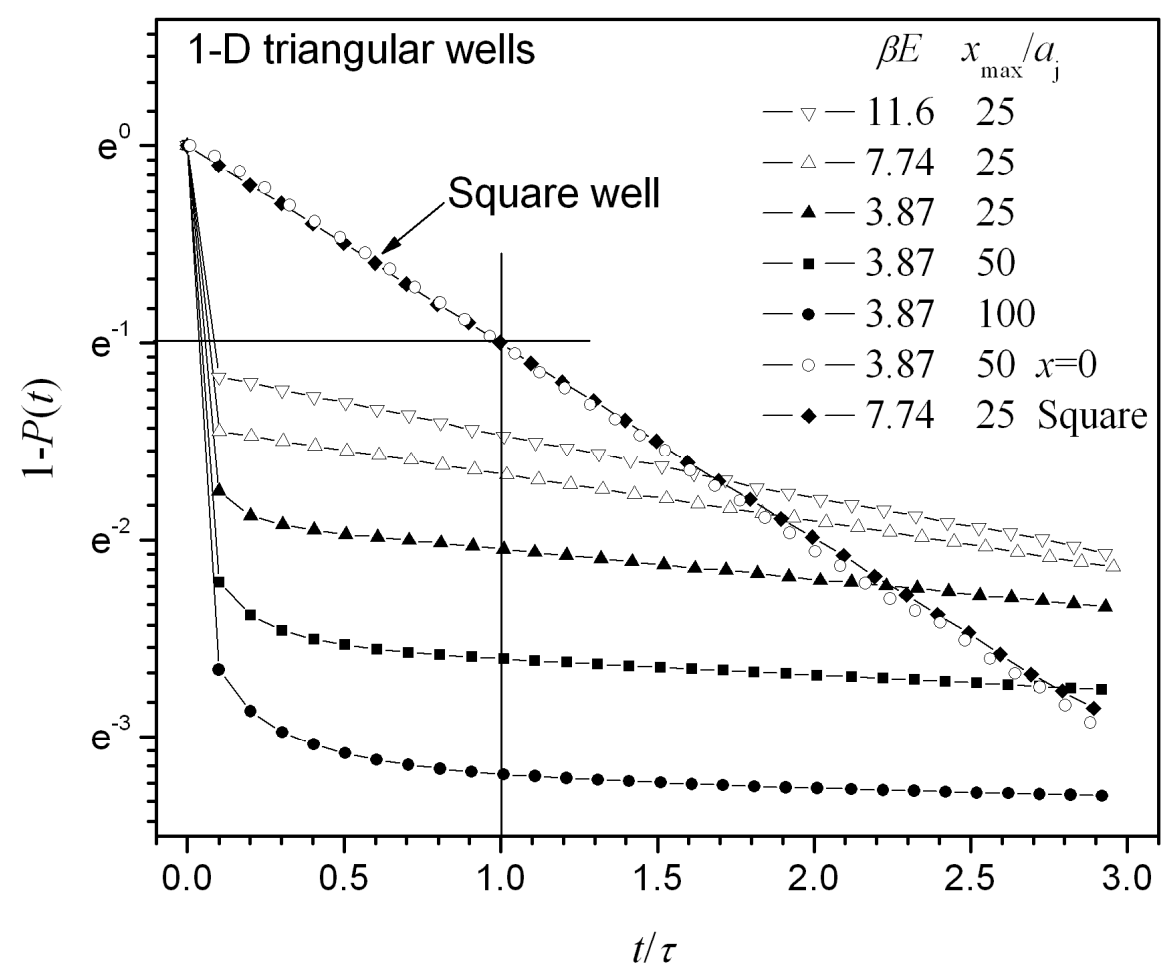

Figure 1. Probability that a dissociation event occurs after time $t$ for 1-D triangular wells of different width and depth, calculated by MC. 


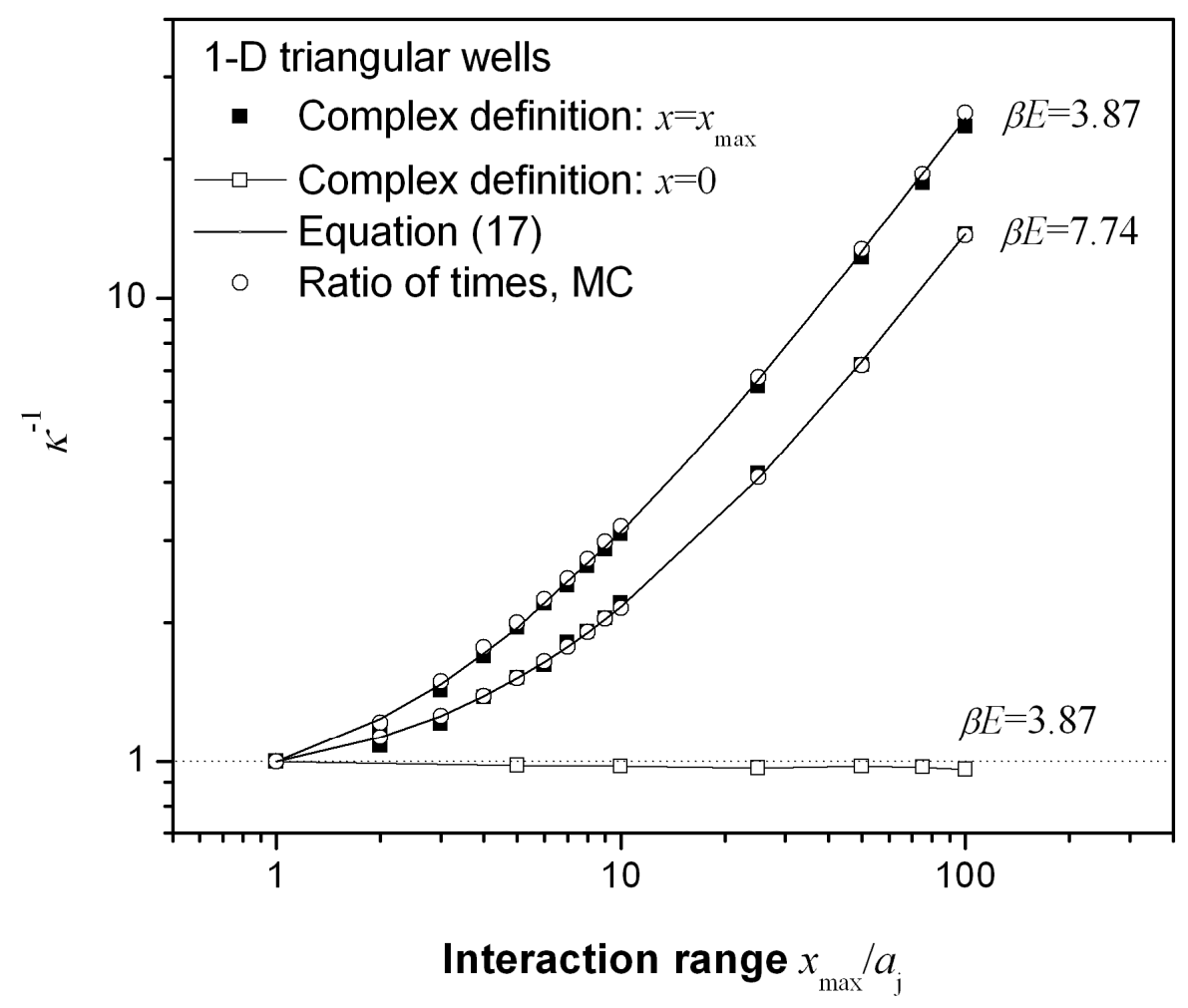

Figure 2. The dependence of $\kappa^{-1}$ and the ratio of mean dissociation times for two complex definitions on the interaction range calculated by $\mathrm{MC}$ results for 1-D triangular wells. 


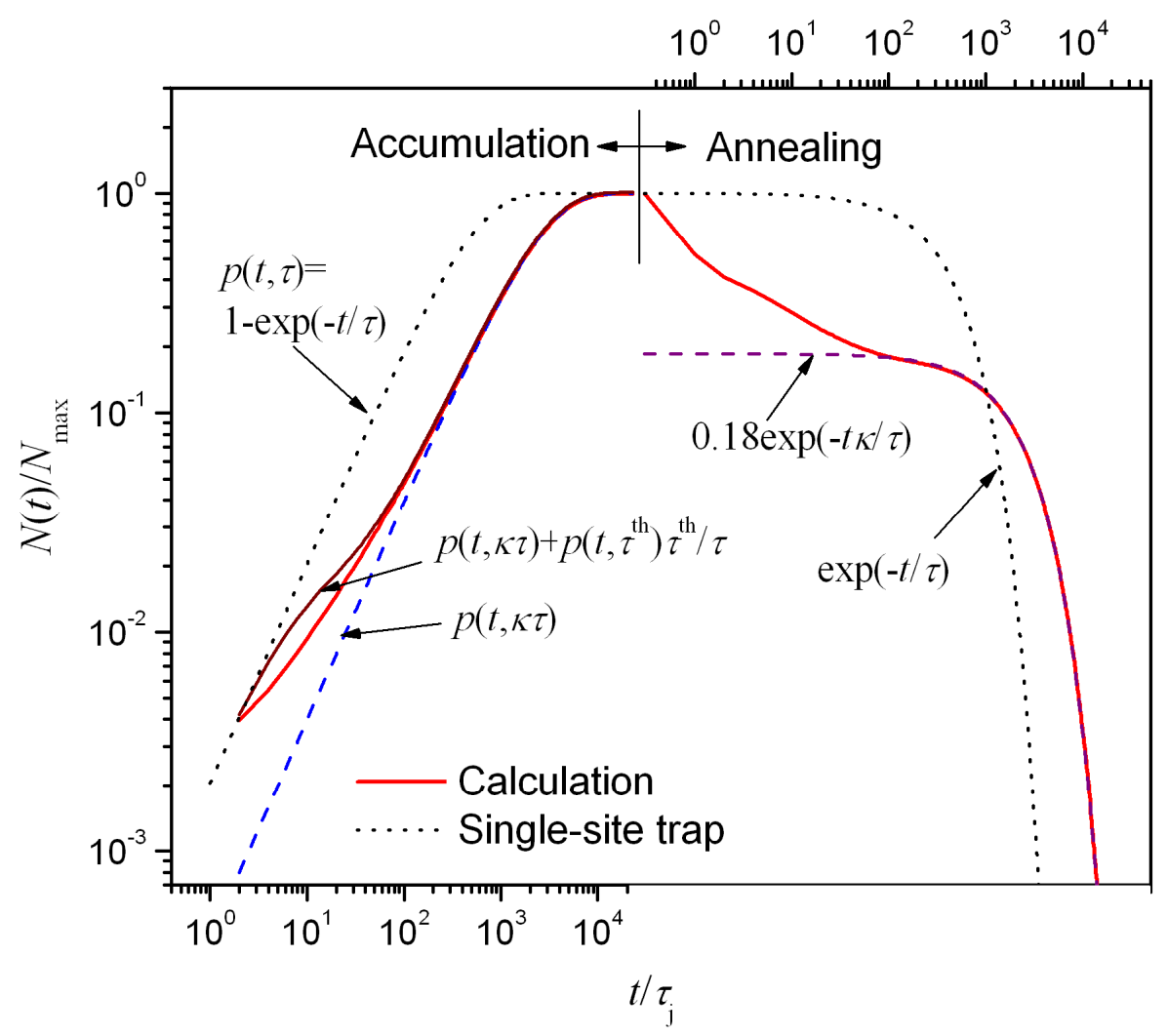

Figure 3. Time dependence of the number of complexes of migrating particles with 1-D triangular wells during steady complex formation and annealing. 